\title{
Recovery of the blood-aqueous barrier after cataract
}

\section{surgery}

\author{
V M G Ferguson, D J Spalton
}

\begin{abstract}
Following extracapsular cataract and posterior chamber implant surgery the sequential recovery of the blood-aqueous barrier was measured by anterior segment fluorophotometry. Postoperatively $49(69.0 \%)$ out of 71 eyes (71 patients) had recovered at a uniform rate, re-establishing a normal blood-aqueous barrier by the end of the three-month study. In these eyes recovery of the blood-aqueous barrier was unaffected by the use of preoperative indomethacin, the surgeon, the type of section, or the type of fixation of the implant. In eyes recovering normally after cataract surgery the rate of recovery of the bloodaqueous barrier can be expressed by $a$ in the equation $a=(y-b) / x$, in which $y$ is the logarithm of the anterior chamber fluorescence, $x$ is the time after surgery, and $b$ is a constant for each patient which is the anterior chamber fluorescence measured immediately after surgery. This normal rate of recovery provides a baseline from which to assess surgical technique or postoperative medication.
\end{abstract}

The results of cataract surgery and visual recovery vary in the postoperative period, but in the long term they are highly successful, with vision of $6 / 12$ or better in over $95 \%$ of patients. Failure in otherwise normal eyes is related to poor placement of the implant, residual soft lens matter, posterior capsular thickening, uveitis from surgical trauma or chronic chafing of uveal tissue, low grade infection, or cystoid macular oedema. Breakdown of the blood-ocular barriers (both the blood-aqueous barrier anteriorly and the blood-retinal barrier posteriorly) may occur in these conditions and has been implicated in the pathogenesis of many of these problems. ${ }^{12}$

Anterior segment fluorophotometry is an objective, reproducible, and simple technique ${ }^{3}$ for measuring the degree and duration of breakdown of the blood-aqueous barrier (BAB) and was used in this study to follow the sequential recovery of the $B A B$ in 71 patients for the first three months after surgery, with the aim of studying the factors that influence the immediate postoperative recovery of the eye following routine extracapsular cataract extraction and insertion of a posterior chamber implant.

Medical Eye Unit, St Thomas's Hospital, London SE1 7EH

V M G Ferguson

D J Spalton

Correspondence to:

D J Spalton, FRCS.

Accepted for publication

16 August 1990
Material and methods

Approval was obtained from the West Lambeth Health Authority Ethical Committee for this study. Patients had no severe cardiovascular or respiratory disease or severe allergy and were willing to co-operate with intravenous fluorophotometry. There were 71 patients (71 eyes studied) of which 28 were males and 43 females (58 Caucasian, 10 Negro, 3 Asian), and all were aged between 43 and 81 (mean 66.4 years, SD 9.4). Extracapsular cataract surgery with insertion of a posterior chamber implant was carried out by three experienced surgeons, each performing his routine technique, the differences between them being in position of the section and fixation of the implant (either in the ciliary sulcus or in the capsular bag). Patients were not on any topical or systemic anti-inflammatory drugs prior to surgery apart from those prescribed preoperative topical indomethacin, and no subconjunctival steroids were given after surgery. The operative details recorded were the surgeon, the type of section, the position of the implant (either in the ciliary sulcus or in the capsular bag), and the amount of trauma, if any, to the iris.

Within seven days of surgery and at one, two, and three months postoperatively a record was made of the clinical findings, and anterior segment fluorophotometry was performed. All patients received fluorescein from the same batch to avoid manufacturing variations in available fluorescence. Fluorescein angiography was performed (sodium fluorescein $20 \% \mathrm{w} / \mathrm{v}, 14 \mathrm{mg}$ / $\mathrm{kg}$, intravenously) so that optic disc leakage and angiographic cystoid macular oedema could be recorded, and one hour later anterior chamber fluorophotometry scans were made with the Fluorotron Master (Coherent Radiation). The fluorophotometer measures anterior chamber (AC) fluorescence as the fluorescence of an equivalent concentration of free fluorescein in $\mathrm{ng} / \mathrm{ml}(=\mu \mathrm{g} / \mathrm{l})$.

The normal level of AC fluorescence for eyes with intact BABs was defined from our other

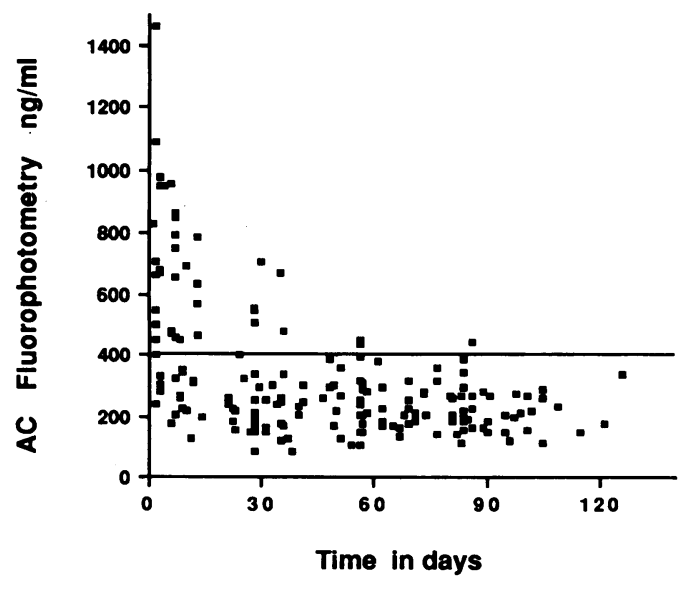

Figure 1 Scatter graph of AC fluorescence with time for all 49 eyes which recovered uneventfully. There are high levels immediately after surgery, which fall during the first month. At three months $69 \%$ of eyes had levels of AC fluorescence below $400 \mathrm{ng} / \mathrm{ml}(\mu \mathrm{g} / \mathrm{l})$. 


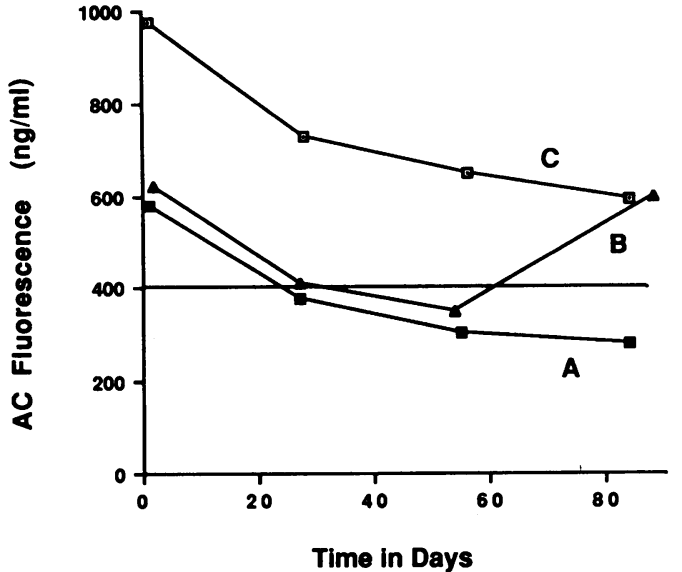

Figure 2 AC fluorescence falls with time, shown by the results from three patients. A has recovered normal levels of AC fluorescence by three months. B appears to recover initially but has an unexpected rise in AC fluorescence at three months. $\mathrm{C}$ continues to have prolonged breakdown throughout the study. (Horizontal line indicates upper limit of normal leakage, $400 \mathrm{ng} / \mathrm{ml}=\mu \mathrm{g} / \mathrm{l}$.)

work on healthy volunteers of the same age as those in the cataract study ( 36 eyes, mean age 61 years). In that study a mean AC fluorescence of $301 \mathrm{ng} / \mathrm{ml}( \pm 108$ mean SD) with a range of 140 $\mathrm{ng} / \mathrm{ml}$ to $560 \mathrm{ng} / \mathrm{ml}(=\mu \mathrm{g} / \mathrm{l})$ was measured one hour after giving intravenous sodium fluorescein $14 \mathrm{mg} / \mathrm{kg}$. In this study, because we were interested in those with normal recovery, patients were taken to have re-established normal BABs by three months if the final fluorophotometry reading was less than $400 \mathrm{ng} /$ ml (therefore excluding those with excess leakage).

The 49 patients with normal recovery of the BAB were divided into groups according to the operating surgeon, the type of section (limbal or corneal), the position of the implant (sulcus or bag fixation), and whether topical indomethacin was used.

\section{Results}

The trend was for high leakage of fluorescent metabolites into the AC during the first postoperative week, with a steady fall in AC fluorescence during the following three months. By this time 49 eyes out of the total of $71(69 \cdot 0 \%)$ had normal levels of AC fluorescence below $400 \mathrm{ng} /$ $\mathrm{ml}(\mu \mathrm{g} / \mathrm{l})($ Fig 1$)$. Of the remaining eyes 14 $(19 \cdot 7 \%)$ still had high levels at three months and $8(11 \cdot 3 \%)$ recovered initially, but subsequently had high levels. Typical examples are illustrated in Figure 2. The reasons for failure to recover the $\mathrm{BAB}$ in these patients are the subject of a subsequent report.

Sequential fluorophotometry measurements of the 49 eyes which had re-established intact blood-aqueous barriers were interpreted by calculating the rate of recovery of each patient. This was represented mathematically by the gradient $a$ of the line $y=a x+b$, where $y$ was the $A C$ fluorescence on a logarithmic scale and $x$ the time in days after surgery. There was a good correlation between the AC fluorescence and time for surgery when the best-fit linear graph was drawn through the four fluorophotometry measurements for each of the 49 patients. The mean

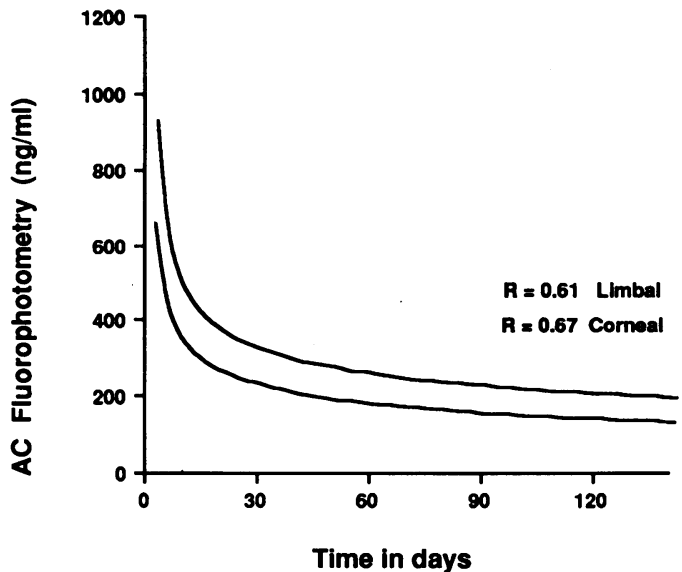

Figure 3 Rates of recovery of the eyes with limbal compared with corneal sections, shown by the change in AC fluorescence with time after surgery. $R=$ coefficient of correlation for the best-fit curve through the data for limbal and corneal groups. Immediately after surgery the group with limbal sections have higher anterior segment leakage, but at three months both groups have re-established normal BABs. $(n g / m l=\mu g / l$.

correlation coefficient for all 49 patients was 0.72 (SD 0.27). This mathematical expression of the recovery of eyes following surgery demonstrated that the $B A B$ recovered to normal in a linear fashion.

These 49 patients were analysed further by grouping them so that comparisons could be made between the rate of recovery of eyes operated on by different surgeons and using different surgical techniques. Statistical comparison of the groups of patients was made by the Mann-Whitney U test.

The first two groups compared were those with sulcus placed implants but operated on by two different surgeons (Fig 3). Group A (nine eyes by surgeon 1) had a limbal section and group B (11 eyes by surgeon 2 ) had a corneal section. There was no statistically significant difference between the two groups (Mann-Whitney $U$ test, $p>0 \cdot 1)$. There was a tendency for the eyes with limbal sections to have slightly higher levels of AC fluorescence initially, but at three months there was no significant difference between them. Apart from the type of section, both surgeons used similar techniques and the eyes showed comparable postoperative progress, final visual results, and final recovery of the bloodaqueous barrier, so all 20 eyes from these two surgeons were grouped as the 'sulcus group'.

The sulcus group and those with bag fixation of the implant ( 15 eyes by surgeons 1 and 2 ) were compared (Fig 4). Best fit curves show similar rates of recovery, falling to within $400 \mathrm{ng} / \mathrm{ml}$ $(\mu \mathrm{g} / \mathrm{l})$, indicating recovery of the BAB by three months. The eyes with implants in the capsular bag had lower leakages initially $(p>0 \cdot 1)$ but by three months they had a slightly higher AC fluorescence than those with sulcus fixed implants, but this was not statistically significant (Mann-Whitney U test, $\mathrm{p}>0 \cdot 1$ ). Figure 5 shows the results of those with bag fixed implants, one group had no indomethacin ( 15 eyes by surgeons 1 and 2), and the other was treated with topical indomethacin preoperatively ( 11 eyes by surgeon $3)$. There was no significant difference between the mean rate of recovery for each group (MannWhitney U test, $\mathrm{p}>0 \cdot 1$ ). 
Figure 4 Rates of recovery of eyes comparing implant fixation. $R=$ coefficient of correlation for the best-fit curve through the data for sulcus and bag groups. Initially those with sulcus fixation have greater $A C$ leakage than those with bag fixation, but at three month there was no statistically significant difference between them. $(n g / m l=$ $\mu g / l$.)

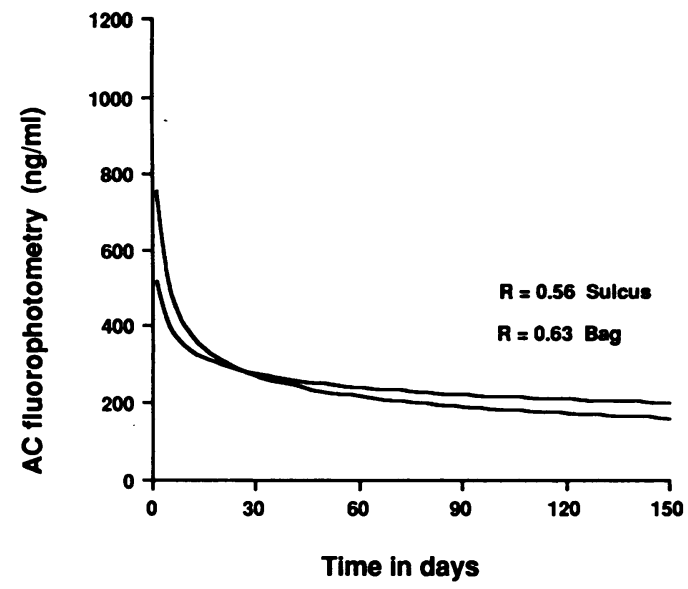

Analysis of the individual slopes of recovery did not show any evidence that the rate of recovery varied between the 49 eyes. The mean gradient was -0.004 (SD 0.003). That is, the eyes that recovered to normal all recovered at the same rate, the duration to recovery being solely determined by the degree of initial damage to the BAB (Fig 6).

\section{Discussion}

In cataract surgery the blood-aqueous barrier, which consists of the endothelium of iris blood vessels and the non-pigmented ciliary epithelium, is susceptible to surgical trauma at the time of operation, and this damage can either lead to recovery quickly or be prolonged for weeks or months after surgery. Several authors have looked at recovery of the $B A B$ after cataract surgery, but this is the only sequential study of recovery of a cohort of patients with time. This report looks at those eyes $(69 \%)$ which recovered a normal BAB within three months and at whether the speed of recovery was influenced by factors such as a limbal or corneal section, position of the implant in the bag or ciliary sulcus, the use of preoperative topical indomethacin, or the operating surgeon. We found no difference in the rate of recovery of the $\mathrm{BAB}$ in the first three months postoperatively from any of these factors.

Recovery of the BAB has been followed by iris angiography, which gives a qualitative assessment, ${ }^{4}$ but anterior segment fluorophotometry enables a quantitative measure of recovery of the BAB. There are various ways of interpretating the data from the fluorophotometer. Miyake $e t a l^{5}$ used ratios comparing the aqueous

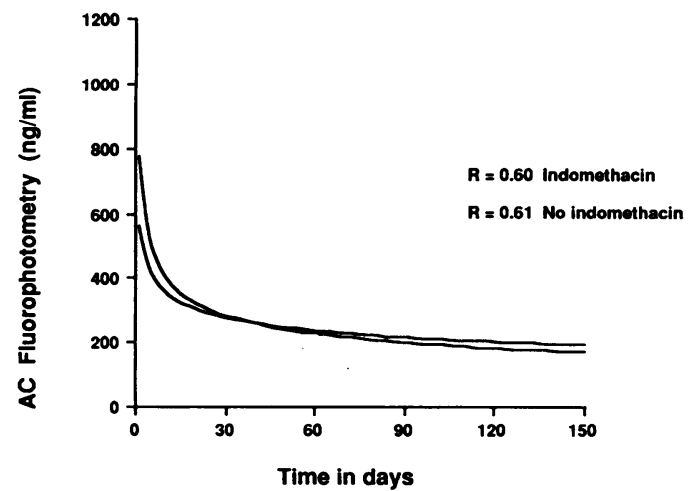

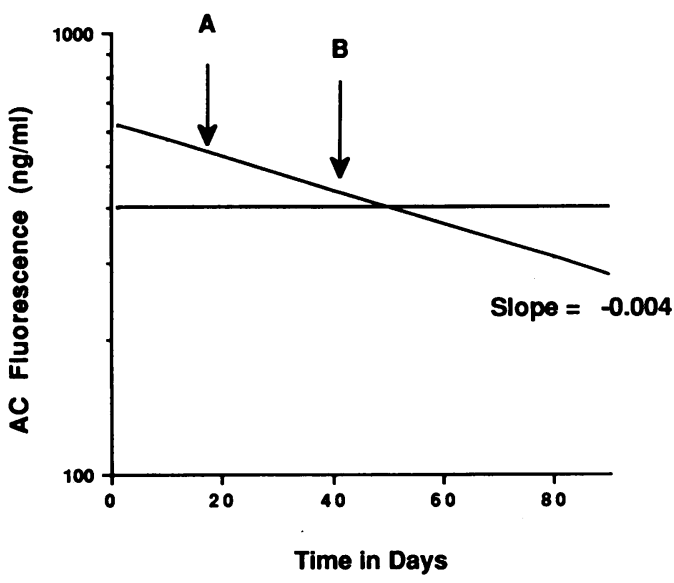

Figure 6 Normal eyes recover their $B A B$ in a linear fashion despite different amounts of surgical damage to the $B A B$, provided their subsequent recovery is uneventful. For example, if a patient had $B A B$ damage $(\mathrm{A})$ postoperatively, the eye would take longer to recover than a patient with less damage (B), because the rate of recovery is the same for both of them. The slope for recovery is linear, so only one measurement is needed postoperatively to predict the time for normal restoration of the BAB. $(n g / m l=\mu g / l$. $)$

fluorescein concentration in the operated eye to the aqueous fluorescein concentration in the fellow eye. Sanders $e t a l^{2}$ looked at the pre- and postoperative levels and compared the percentage change in aqueous fluorescence in the operated eye with that in the fellow unoperated eye. Leisegang $e t a l^{6}$ calculated the ratio of aqueous to plasma fluorescence after giving oral fluorescein to produce an index of BAB function. Sawa et $^{\mathrm{al}}{ }^{7}$ compared ratios of aqueous to plasma fluorescence after giving intravenous fluorescein to produce an index of BAB function. Araie $e t a l^{8}$ calculated the transfer coefficient of diffusion after giving intravenous fluorescein, and Menerath et $a l^{9}$ calculated a transfer coefficient after giving oral fluorescein.

There are potential problems with all of these methods of measuring recovery of the $\mathrm{BAB}$. The consensual response to surgery, in which there may be breakdown of the $\mathrm{BAB}$ in the fellow eye, ${ }^{10}$ means that the fluorescence measured in the fellow, unoperated eye cannot be used as a normal level against which to compare changes in the operated eye. Comparisons between preand postoperative measurements are complicated by the glare effect from autofluorescence of the cataractous lens. This artefact disappears when the cataract has been removed and an implant inserted (implants do not autofluoresce).

In order to calculate indices of permeability or diffusion assumptions have to be made, firstly, about fluorescein metabolism and its pharmacokinetics in the blood and in the eye (there are varying amounts of free and protein bound fluorescein and fluorescein glucuronide); and, secondly, the eye is taken as standard with spherical surfaces, a given area of iris and a given AC volume. These physiological formulae are not always valid in real patients, particularly in the inflamed eye. We took the plateau levels of $\mathrm{AC}$ fluorescence which we have done in previous studies $^{3}$ (Ambrose VMG, et al, personal communication). They are simple to measure, with a reproducibility of $87 \%$ (comparing measurements on the same subjects on two separate visits, the nested analysis of variance showed an 
intrapair correlation coefficient of $r=0.871$ ), and they provide an absolute measure of the degree of breakdown of the BAB which can be compared between patients.

In eyes with uncomplicated surgery we found that the $\mathrm{BAB}$ recovered rapidly during the first six weeks, and at three months a normal intact blood-aqueous barrier was demonstrated by stable plateau levels of AC fluorescence in $69.0 \%$ of eyes. But $31 \%$ of eyes failed to re-establish normal BABs. These included $19 \cdot 7 \%$ (14 eyes) which still had high AC levels at three months and $11 \cdot 3 \%$ (eight eyes) which appeared to recover normally at first but then had abnormally high levels. Interestingly, this proportion correlates well with the histopathological findings by Champion et $a l^{11}$ who found that $34 \%$ of postmortem eyes examined between eight months and 10 years after implant insertion showed evidence of cyclitis and iritis, and it suggests that inflammatory processes underlie failure to recover $\mathrm{BAB}$ function in most cases.

Other workers have also looked at the duration of breakdown and influence of implant position on recovery of the BAB. Sanders $e t a l^{2}$ showed that in eyes with uncomplicated surgery and postoperative subconjunctival and topical steroids the BAB was re-established by three months after surgery. Sawa et $\mathrm{ll}^{7}$ used an index of BAB function in the early postoperative period (six days after operation) and found that insertion of a posterior chamber implant caused no increase in breakdown of the blood-aqueous barrier in comparison with eyes with extracapsular extraction without any implant. In a French study Menerath $e t \mathrm{al}^{9}$ gave oral fluorescein, calculated a transfer coefficient, and found no difference in $\mathrm{BAB}$ dysfunction with and without an AC implant after intracapsular cataract surgery. They also gave oral piroxicam before and after surgery and found reduced BAB breakdown three days after surgery but no difference at two months in comparison with an untreated group. These results suggest that, in normal eyes with uncomplicated surgery, recovery of the BAB in the initial postoperative period is not influenced by either the presence or absence of an implant or its position in the bag, sulcus, or anterior chamber and that the changes in the BAB reflect a normal healing and recovery process in the iris and ciliary body.

While in the short term implant fixation may be unimportant in terms of recovery of the $\mathrm{BAB}$, in the longer term there may well be significant implications. Miyake et $a l^{512}$ looked at eyes with uncomplicated extracapsular cataract surgery (average follow-up of one year after operation) and found that for posterior chamber implants those with sulcus fixation leaked more than those with bag fixation. They also showed that eyes with bag fixed implants had comparable leakage to eyes without implants. After a longer followup of three years there was no difference in the leakage between aphakic eyes and those with bag or sulcus implants. These results imply that the advantage of bag to sulcus fixation is the prevention of uveal chafing or erosion, which becomes obvious or important only on medium to long term follow-up.

In any study comparing the advantages of bag to sulcus fixation of the implant in the posterior chamber it is necessary to know that the implant is actually where the surgeon intended to put it. In reality it is extremely difficult to assess this accurately, particularly when $7 \mathrm{~mm}$ diameter implants are used, as it is not easy to be certain that the implant is in the bag. It may be difficult to dilate the pupil to greater than this diameter, and even if the optic is contained in the capsule the haptics may have slipped out or eroded through it. In a post-mortem study Champion $e t$ $a l^{11}$ and McDonnell et al1 ${ }^{13}{ }^{14}$ looked at 65 postmortem eyes. Only $5 \%$ of eyes had both loops in the capsular bag, 54\% had one loop in the bag, and $42 \%$ had both loops out of the bag. Of the eyes with only one loop in the bag, $77 \%$ had the lower loop in and $23 \%$ had the superior loop in the bag. Overall, they found that erosion of the ciliary body was related to the size of the implant and elasticity of the haptics rather than the surgical placement or the length of time the implant had been in the eye. From this work one must accept that there must be unavoidable doubts about the true site of implant fixation in any clinical study unless the ciliary body itself can be directly visualised.

Topical treatment with indomethacin preoperatively (one drop twice daily for two days preoperatively) did not appear to influence recovery of the $B A B$. The role of topical or systemic prostaglandin inhibitors in cataract surgery has been studied extensively with mixed results. Sanders and Kraff ${ }^{15}$ measured breakdown of the BAB following uncomplicated extracapsular cataract and implant surgery in which patients were given topical indomethacin preoperatively and for two weeks postoperatively. At two weeks fluorophotometry with oral fluorescein was performed and showed reduced BAB breakdown, indicating that indomethacin had an additive or synergistic effect with topical dexamethasone. Pre- and postoperative topical indomethacin in addition to steroids shortened the recovery time of the blood-aqueous barrier from 12 to five weeks but did not influence the integrity of the BAB immediately after surgery.

Miyake $^{12}$ performed fluorophotometry (after giving intravenous fluorescein) four weeks after cataract surgery by phacoemulsification and showed less AC fluorescence in those patients treated with topical indomethacin postoperatively, but at two years there was no difference between the treated and placebo groups. Araie $e t$ $a l^{8}$ used fluorophotometry to measure the transfer coefficient of eyes after intracapsular cataract surgery and found that topical indomethacin inhibited barrier disruption at six days after surgery. These results suggest that indomethacin has a beneficial early action but has less influence in the long term. A possible explanation is that the effects of prostaglandin inhibitors are secondary to maintaining pupillary dilatation (which they do very effectively). That is, they may well act by reducing surgical trauma and manipulation to the iris at the time of operation rather than by direct action on the BAB.

In addition to finding a similar recovery of breakdown of the $\mathrm{BAB}$ in all the eyes we have found a uniform rate of return to normality in those eyes that had recovered their BAB by three 
months independently of surgeon, type of section, placement of implant, or use of preoperative indomethacin. If fluorophotometry is plotted on a logarithmic scale against time, the rate of recovery can be described by the equation $a=(y-b) / x$, where $y$ is the AC fluorescence and $x$ is the time after surgery. This implies that eyes with higher levels of $\mathrm{BAB}$ dysfunction immediately after surgery will recover at the same rate as those with less damage but will take longer to do so, provided this 'normal' recovery process is not interfered with by secondary inflammatory processes. One measurement should be necessary to enable prediction of the duration of recovery of the $\mathrm{BAB}$ and to provide a baseline against which changes in surgical technique or postoperative medication could be assessed (Fig 6 ).

This study was supported by the Iris Fund for Prevention of Blindness Ltd, London.

1 Apple DJ, Craythorn JM, Randall JO, et al. Anterior segment complications and neovascular glaucoma following complications and Ophthalmology 1984; 91: 403-19.

2 Sanders DR, Kraff MC, Lieberman HL, Peyman GA, Tarabishy S. Breakdown and reestablishment of blood aqueous barrier with implant surgery. Arch Ophthalmol 1982; 100: 588-90.

3 Fearnley IR, Spalton DJ, Smith SE. Anterior segment fluorophotometry in acute anterior uveitis. Arch Ophthalmol 1987; 105: 649-56.

4 Easty D, Dallas N, O'Malley R. Aphakic macular oedema following prosthetic lens implantation. $\mathrm{Br} \mathcal{F}$ Ophthalmol 1977; 61: $321-6$

5 Miyake K, Asakura M, Kobayashi H. Effect of intraocular lens fixation on the blood-aqueous barrier. Am $\mathcal{F}$ Ophthalmol 1984; 98: 451-5.

6 Liesegang TJ, Bourne WM, Brubaker RF. The effect of cataract surgery on the blood-aqueous barrier. Ophthalcataract surgery on the

7 Sawa M, Sakanishi Y, Okuno Y, Shimizu H. Posterior chamber lens implantation and anterior segment arrier function: a fluorophotometric study. $\mathcal{f} p n \mathcal{F}$ Ophthalmol 1988; 32: 297-303.

8 Araie M, Sawa M, Takase M. Effect of topical indomethacin on the blood-aqueous barrier after intracapsular extraction of senile cataract: a fluorophotometric study. $7 \mathrm{pm} f$ Ophthalmol 1981; 25: 237-47.

9 Menerath JM, Coulangeon LM, Al-Odeh A. Evaluation fluorophotometrique des barrières hématooculaires dans la chuorophotometrique des barrières hématooculaires dans

10 Miyake K, Asakura M, Maekubo K. Consensual reactions of human blood-aqueous barrier to implant operations. Arch Ophthalmol 1984; 102: 558-61.

11 Champion R, McDonnell PJ, Green WR. Intraocular lenses. Histopathologic characteristics of a large series of autopsy eyes. Surv Ophthalmol 1985; 30: 1-32.

12 Miyake K. Fluorophotometric evaluation of the blood-ocular barrier function following cataract surgery and intraocular lens implantation. $f$ Cataract Refract Surg 1988; 14:560-8.

13 McDonnell PJ, Green WR, Champion R. Pathologic changes in pseudophakia. Semin Ophthalmol 1986; 1: 80-103.

14 McDonnell PJ, Green WR, Maumenee AE, Iliff WJ. Pathology of intraocular lenses in 33 eyes examined postPathology of intraucular lenses in 33 eyes

15 Sanders DR, Kraft M. Steroidal and non-steroidal antiinflammatory agents. Effect on post surgical inflammation and blood-aqueous humor breakdown. Arch Ophthalmol 1984; 102: 1453-6. 